\title{
Explicit solutions of wall jet flow subject to a convective boundary condition
}

\author{
Ammarah Raees ${ }^{1 *}$, Hang Xu ${ }^{1}$ and Muhammad Raees-ul-Haq ${ }^{2}$
}

${ }^{*}$ Correspondence:
ammarah@sjtu.edu.cn
'State Key Lab of Ocean
Engineering, School of Naval
Architecture, Ocean and Civil
Engineering, Shanghai Jiao Tong
University, Shanghai, 200240, China
Full list of author information is
available at the end of the article

available at the end of the article

\begin{abstract}
In this paper, an analysis is made on the laminar jet flow and heat transfer of a copper-water nanofluid over an impermeable resting wall. With the homogeneous model (Maïga et al. in Int. J. Heat Fluid Flow 26(4): 530-546, 2005), the Navier-Stokes equations describing this heat fluid flows are reduced to a set of differential equations via similarity transformations. An implicitly analytical solution overlooked in previous publications is discovered for the velocity distribution. We further present the explicit solutions with high precision for both the velocity and the temperature distributions. A mathematical analysis shows that those explicit solutions have exponential behaviors at far field. Besides, the effects of the volumetric fraction parameter $\phi$ and the dimensionless heat transfer parameter $\gamma$ on the velocity and temperature profiles, as well as on the reduced local skin friction coefficient and the reduced Nusselt number, are examined in detail.
\end{abstract}

Keywords: jet flow; convective heat transfer; exponential behavior; nanofluid flow; convective boundary condition; explicit solutions

\section{Introduction}

In an actual environment, the turbulent wall jets are dominant. While the laminar ones still attract many researchers owing to their numerous practical and potential applications including the cooling systems for the central processing units of laptops, spray-paint processing for vehicles or buildings, downwards-directed jets from a vertical-take-off aircraft spreading out over the ground, cooling jets over turbo-machinery components, sluice gate flows, and so on. The boundary layer approximation is used commonly as an effective approach for simplification of the laminar wall jet problems. The corresponding similarity (or non-similarity) solutions were found to be adequate for the prediction of their behaviors. Among those studies of the laminar wall jet, Glauert [1] was the first to use the name of wall jet for the description of such flows. By means of the similarity method, he reduced the two-dimensional Navier-Stokes equations to an ordinary differential equation and then obtained a well-known implicit analytical solution. Merkin and Needham [2] and Needham and Merkin [3] extended Glauert's problem [1] to the cases that both the wall moving and the wall blowing/suction are allowed. They then concluded that if wall moving is introduced, the similarity solution could be only possible when an appropriately lateral suction is applied through the moving surface. Magyari and Keller [4] confirmed Merkin and Needham's conclusion [2] that no similarity solution of Glauert's type exists for the wall jet flowing over a resting surface in the presence of suction and/or injection.

(c) 2014 Raees et al.: licensee Springer. This is an Open Access article distributed under the terms of the Creative Commons Attribution License (http://creativecommons.org/licenses/by/2.0), which permits unrestricted use, distribution, and reproduction in any medium, provided the original work is properly cited. 
Instead, they found that a particular kind of solution with algebraically decaying behavior is in existence for the wall suction case. While Cohen et al. [5] argued that the solutions of Glauert's type could be possible for the resting surface case when the suction/injection velocity is proportional to $x^{-b}$ for $1<b<3$. Xu et al. [6] further found that except for the solutions given by Cohen et al. [5], an infinite number of solutions of an algebraical nature exist for the considered case. For heat transfer problems, Magyari et al. [7] made an analysis on heat transfer characteristics of a boundary layer flow driven by a power-law shear at far field over an impermeable flat surface. Mathematically, their problem is equivalent to a wall jet flowing over a heated flat surface. They then presented both the analytical and the numerical solutions for the special cases of the isothermal and of the adiabatic flat plate. Cossali [8] considered a forced convection thermal boundary layer over an impermeable flat surface driven by an outer power-law shear. He obtained a family of similarity solutions for various values of the exponent of the decaying exterior velocity profile and the exponent of the power-law prescribing the thermal condition on the wall. Very recently, Fan and Xu [9] extended Magyari et al.'s [7] problem to the case that the plate is permeable. They found that both exponentially and algebraically decaying solutions could be possible when the suction is applied through the wall. They then presented a family of solutions covered for various power-law distributions of the outer velocity and the wall temperature both analytically and numerically.

As a new generation heat transfer fluids, nanofluids have received more and more attention for the reason that they possess a better thermal conductivity than that of the traditional heat transfer fluids. Many researchers have made great efforts to investigate the heat fluid flow in nanofluids under various circumstances since the heat transfer intensification due to dilute nanofluids could provide opportunities for a number of innovative applications in industrial sectors such as transportation, power generation, micromanufacturing, thermal therapy for cancer treatment, chemical and metallurgical sectors, as well as heating, cooling, ventilation, and air-conditioning. Several approaches have been suggested for modeling nanofluids. Typical models include the homogeneous model [10, 11], the dispersion model [12], the Buongiorno model [13] and so on. Note that there are controversies about the validity and applicability of those nanofluids models for the prediction of the behavior of nanofluids. Readers are referred to [14-18] for more details. Among those models, the homogeneous model is the most popular one since it is very convenient to extend the conventional conservation equations for pure fluids to nanofluids. As a result, all traditional heat transfer correlations regarding the computation of thermophysical properties could be suitable for nanofluids as well. This model is only valid for dilute nanofluids with a similar behavior to Newtonian fluids. When the concentration of the nanofluids grows high, the nanofluids no longer have the nature of Newtonian fluids, but exhibit behaviors like non-Newtonian fluids. In such a situation, this model cannot be used for modeling nanofluids. Fortunately, it has been found that, even for dilute nanofluids, the heat transfer enhancement can still be improved significantly as compared with the traditional heat transfer fluids. With the homogeneous model, some investigations have been done towards understandings of the heat transfer characteristics of nanofluids. For examples, Bachok et al. [19] investigated a boundary layer flow and heat transfer due to a rotating disk immersed in a nanofluid. Their theoretical results show that one can distinguish how the addition of nanoparticles into pure fluids can improve the heat transfer capability of the fluids even if the amount of added nanoparticles is small. Rohni [20] 
made an analysis for a viscous nanofluid flow and heat transfer over an unsteady shrinking flat surface. Considering three kinds of nanofluids, including $\mathrm{Cu}$-water, $\mathrm{Al}_{2} \mathrm{O}_{3}$-water and $\mathrm{TiO}_{2}$-water, they concluded that the nanoparticle volume fraction parameter (which is associated with the concentration of nanofluids) and types of nanofluid play a key role for determination of the flow behavior. Similar conclusions were, respectively, given by Yacob et al. [21] and Vajravelu et al. [22] via their investigations about the flow and heat transfer of nanofluids past a wedge and over a flat surface.

In previous studies about the heat transfer problems in the boundary layer, the isothermal wall condition and the constant wall heat flux condition were frequently used since they possess a simple mathematical structure and often admit similarity solutions. Recently, Aziz [23] made an analysis on a thermal boundary layer over a convectively heated flat surface in a uniform stream of fluid. He found that the similarity solutions could also be possible if the convective heat transfer associated with the hot fluid on the lower surface of the plate is inversely proportional to the square root of the distance along the wall. Ishak [24] extended Aziz's work [23] by introducing the effects of suction and injection through the flat surface and then presented similarity solutions with the same heat transfer coefficient. Aziz and Khan [25] discovered similarity solutions for a free convective flow of a nanofluid about a vertical surface with a convective boundary condition by assuming that the convective heat transfer coefficient for the hot fluid varies inversely with the fourth root of the distance along the vertical wall. Makinde and Aziz [26] derived a similarity solution for a hydromagnetic mixed convection flow past a convectively heated vertical plate embedded in a porous medium with the convective heat transfer coefficient for the hot fluid being a constant. Hayat et al. [27] noticed that the convective boundary condition could also be applied to derive similarity solutions for non-Newtonian fluids. They then obtained the similarity solutions for the problem of the flow and heat transfer of an Eyring Powell fluid over a continuously moving surface in the presence of a free stream velocity. It should be noted to this end that investigations of flow and heat transfer problems with convective boundary conditions are very attractive and unique, since they are more realistic and practically useful than those with commonly used conditions of a constant surface temperature or constant heat flux.

The aim of this paper is to investigate the laminar nanofluid flow and heat transfer due to a jet spreading out over a convectively heated flat surface. The homogeneous model will be introduced to the boundary layer equations for modeling the nanofluid. Similarity solutions of the boundary layer equations will be sought, according to which the forms of the velocity distribution across the jet and the heat transfer coefficient associated with the hot fluid on the lower surface of the plate are assumed, respectively, to vary inversely proportional to the square root and the three-quarter root of the distance along the flat surface from the leading edge. Explicit analytical approximations with high precision will be given for both the velocity and the temperature distributions. Besides, the important quantities of practical interests including the boundary layer thickness, the skin friction coefficient, the Nusselt number, as well as the overall surface heat transfer rate are computed and discussed. To the best of our knowledge this problem has not been considered before and the results are original and new.

\section{Mathematical description}

Consider a two-dimensional laminar jet flowing over a flat plate in a viscous nanofluid of temperature $T_{\infty}$. As shown schematically in Figure 1, a jet of fluid spreads out from a 
Figure 1 The physical sketch.

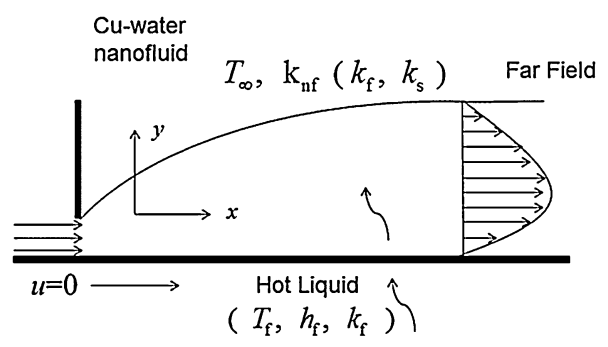

narrow slit to the upper surface of the plate, while the lower surface of the plate is heated or cooled by convection from a fluid of temperature $T_{f}$. With the assumptions that (1) the fluid is incompressible and the flow is laminar, (2) the nanofluid is dilute, (3) the shape of the metal nanoparticles suspended in the fluid is spherical, and (4) the homogeneous model developed by Maïga et al. [11] is employed, the velocity and temperature within the momentum and thermal boundary layers, which develop along the surface, can be written as

$$
\begin{aligned}
& \frac{\partial u}{\partial x}+\frac{\partial v}{\partial y}=0, \\
& u \frac{\partial u}{\partial x}+v \frac{\partial u}{\partial y}=\frac{\mu_{n f}}{\rho_{n f}} \frac{\partial^{2} u}{\partial y^{2}}, \\
& u \frac{\partial T}{\partial x}+v \frac{\partial T}{\partial y}=\alpha_{n f} \frac{\partial^{2} T}{\partial y^{2}},
\end{aligned}
$$

subject to boundary conditions

$$
\begin{aligned}
& u=0, \quad v=0, \quad-k_{n f} \frac{\partial T}{\partial y}=h_{f}(x)\left(T_{f}-T\right) \quad \text { at } y=0, \\
& u \rightarrow 0, \quad T \rightarrow T_{\infty}, \quad \text { as } y \rightarrow \infty .
\end{aligned}
$$

Here the Cartesian coordinates system $(x, y)$ is chosen with the $x$-axis being measured along the plate and the $y$-axis being normal to it, respectively. $u$ and $v$ are the velocity components along the $x$ - and $y$-axes, $T$ is the temperature, $h_{f}(x)$ is the heat transfer coefficient due to $T_{f} ; \mu_{n f}, \rho_{n f}, \alpha_{n f}$, and $k_{n f}$ are, respectively, the viscosity, the density, and the diffusivity and thermal conductivity of the nanofluid, which are given by

$$
\begin{aligned}
& \mu_{n f}=\frac{\mu_{f}}{(1-\phi)^{2.5}}, \quad \rho_{n f}=(1-\phi) \rho_{f}+\phi \rho_{s}, \quad \alpha_{n f}=\frac{k_{n f}}{\left(\rho C_{p}\right)_{n f}}, \\
& \left(\rho C_{p}\right)_{n f}=(1-\phi)\left(\rho C_{p}\right)_{f}+\phi\left(\rho C_{p}\right)_{s}, \quad \frac{k_{n f}}{k_{f}}=\frac{\left(k_{s}+2 k_{f}\right)-2 \phi\left(k_{f}-k_{s}\right)}{\left(k_{s}+2 k_{f}\right)+\phi\left(k_{f}-k_{s}\right)},
\end{aligned}
$$

where $\phi$ is the solid volume fraction of the nanofluid, $\mu$ is the viscosity, $\rho$ is the density, $k$ is the thermal conductivity, $C_{p}$ is the specific heat capacitance, the subscript ' $n f$ ' represents the nanofluid, ' $f$ ' represents the base fluid, and ' $s$ ' represents the nanoparticles, respectively. It should be noted here that the expression for $\mu_{n f}$ is estimated by Brinkman [28] using the existing relations for two-phase mixtures, the expressions for $\rho_{n f}$ and $\left(\rho C_{p}\right)_{n f}$ are given by Xuan and Li [29], and the expression for $k_{n f}$ is approximated by the Maxwell- 
Garnetts model [11] with the assumption that the shape of the nanoparticles is restricted to spherical ones.

We now introduce the following similarity variables:

$$
\psi=\left(v_{f}^{2} \cdot x\right)^{1 / 4} \cdot f(\eta), \quad \eta=\frac{1}{4}\left(v_{f}^{2} \cdot x^{3}\right)^{-1 / 4} \cdot y, \quad \theta(\eta)=\frac{T-T_{\infty}}{T_{f}-T_{\infty}},
$$

where $\psi$ is the stream function defined by $u=\partial \psi / \partial y$ and $v=-\partial \psi / \partial x$, and $v_{f}$ is the kinematic viscosity of the base fluid.

Substituting Eq. (6) into Eqs. (1)-(3), the continuity equation (1) is automatically satisfied, and the momentum equation (2) and the energy equation (3) are reduced to

$$
\begin{aligned}
& \varepsilon_{1} f^{\prime \prime \prime}+f^{\prime \prime}+2 f^{\prime 2}=0, \\
& \frac{\varepsilon_{2}}{P r} \theta^{\prime \prime}+f \theta^{\prime}=0,
\end{aligned}
$$

subject to the boundary conditions

$$
f(0)=0, \quad f^{\prime}(0)=0, \quad \theta^{\prime}(0)=-\gamma[1-\theta(0)], \quad f^{\prime}(\infty)=0, \quad \theta(\infty)=0,
$$

where $\operatorname{Pr}=v_{f} / \alpha_{f}$ is the Prandtl number, $\gamma$ is the reduced heat transfer parameter, and $\varepsilon_{1}$ and $\varepsilon_{2}$ are two constants related to the properties of nanofluids and are given as

$$
\varepsilon_{1}=\frac{1}{(1-\phi)^{2.5}\left[(1-\phi)+\phi \rho_{s} / \rho_{f}\right]}, \quad \varepsilon_{2}=\frac{k_{n f} / k_{f}}{(1-\phi)+\phi\left(\rho C_{p}\right)_{s} /\left(\rho C_{p}\right)_{f}} .
$$

The physical quantities of practical interest are the local skin friction coefficient $C_{f x}$ and the local Nusselt number $N u_{x}$, which are given by

$$
C_{f x}=\frac{\tau_{w}}{\rho_{f} u_{r}^{2}}, \quad N u_{x}=\frac{x q_{w}}{k_{f}\left(T_{w}-T_{\infty}\right)},
$$

where $\tau_{w}=\mu_{n f}(\partial u / \partial y)_{y=0}$ is the wall shear stress, $q_{w}=-k_{n f}(\partial T / \partial y)_{y=0}$ is the wall heat flux, and $u_{r}=(1 / 4) x^{-1 / 2}$ is the reference velocity.

In terms of similarity variables (6), we are able to obtain the non-dimensional expressions for $C_{f x}$ and $N u_{x}$ via Eq. (11) as

$$
C_{f x} R e_{x}^{1 / 2}=\frac{2 f^{\prime \prime}(0)}{(1-\phi)^{2.5}}, \quad N u_{x} R e_{x}^{-1 / 2}=-\frac{k_{n f}}{2 k_{f}} \frac{\theta^{\prime}(0)}{\theta(0)},
$$

where $\operatorname{Re}_{x}=\left(u_{r} x\right) / v_{f}$ is the local Reynolds number.

\section{Analytical solutions}

\subsection{Asymptotic analysis}

Here we check the asymptotic behaviors of the velocity profile $f^{\prime}(\eta)$ and the temperature profile $\theta(\eta)$ in the limit case $\eta \rightarrow \infty$. Due to the boundary conditions (9), for considerably large $\eta$, we have

$$
\lim _{\eta \rightarrow \infty} f^{\prime}(\eta) \rightarrow 0, \quad \lim _{\eta \rightarrow \infty} \theta(\eta) \rightarrow 0 .
$$


Integrating $f^{\prime}(\eta)$ over $\eta$ one time, we obtain

$$
\lim _{\eta \rightarrow \infty} f(\eta) \rightarrow \lambda
$$

where $\lambda$ is an integral constant. For the physical constraint $f^{\prime} \geq 0$, it is readily seen that $\lambda \geq 0$.

For $\eta \rightarrow \infty$, we suppose $f(\eta)$ and $\theta(\eta)$ can be expressed by

$$
f(\eta)=\lambda+F(\eta), \quad \theta(\eta)=\Theta(\eta),
$$

where $F(\eta)$ and $\Theta(\eta)$ are negligibly small.

Substituting Eq. (14) into Eqs. (7) and (8) and then linearizing them, we obtain

$$
\begin{aligned}
& \varepsilon_{1} F^{\prime \prime \prime}+\lambda F^{\prime \prime}=0, \\
& \frac{\varepsilon_{2}}{P r} \Theta^{\prime \prime}+\lambda \Theta^{\prime}=0,
\end{aligned}
$$

which have the explicit solutions

$$
\begin{aligned}
& F^{\prime}(\eta)=C_{1} \exp \left(-\frac{\lambda}{\varepsilon_{1}} \eta\right)+C_{2}, \\
& \Theta(\eta)=C_{3} \exp \left(-\frac{\lambda P r}{\varepsilon_{2}} \eta\right),
\end{aligned}
$$

where $C_{1}, C_{2}$, and $C_{3}$ are integral constants.

Due to Eqs. (13) and (14), it is known that

$$
F^{\prime}=\Theta=0 \quad \text { as } \eta \rightarrow \infty .
$$

With substitution of the boundary condition (19) into Eq. (17), we find $C_{2}=0$, which leads to

$$
F^{\prime}(\eta)=C_{1} \exp \left(-\frac{\lambda}{\varepsilon_{1}} \eta\right) .
$$

It can be seen from Eqs. (20) and (18) that $F^{\prime}(\eta)$ and $\Theta(\eta)$ (hence $f^{\prime}(\eta)$ and $\theta(\eta)$ ) decay exponentially as $\eta \rightarrow \infty$.

\subsection{The implicit solution for $f(\eta)$}

We multiply Eq. (7) by $f(\eta)$ and integrate it from $\eta$ to $\infty$, obtaining

$$
\varepsilon_{1} f^{\prime \prime}-\frac{\varepsilon_{1}}{2} f^{\prime 2}+f^{2} f^{\prime}=0
$$

Multiplying Eq. (21) by $f^{-3 / 2}$ and integrating again, we obtain

$$
\varepsilon_{1} f^{-1 / 2} f^{\prime}+\frac{2}{3} f^{3 / 2}=\text { constant }
$$


It is found here that if $f_{0}(\eta)$ is the solution of Eq. (7), so is $f_{1}(\eta)=A f_{0}(A \eta)$, since for any constant $A, f_{1}(\eta)$ always satisfies the boundary conditions (9). When $A$ varies, the expressions for $\psi$ and $\eta$ change accordingly, and $u$ is precisely the same as that of changing the value of the arbitrary constant velocity $U$ to $U / A^{4}$. Without loss of generality, we choose $f(\infty)=1$ so that the constant in Eq. (22) is equal to $2 / 3$. Write $f=g^{2}$; we then have $f^{\prime}=2 g g^{\prime}$. Therefore Eq. (22) takes the form

$$
\varepsilon_{1} g^{\prime}=\frac{1}{3}\left(1-g^{3}\right)
$$

which has the implicit solution

$$
\eta=\varepsilon_{1}\left[\log \left(\frac{\sqrt{1+g+g^{2}}}{1-g}\right)+\sqrt{3} \arctan \left(\frac{\sqrt{3} g}{2+g}\right)\right] .
$$

By replacing $g$ with $f^{1 / 2}$, Eq. (24) can further be written as

$$
\eta=\varepsilon_{1}\left[\log \left(\frac{\sqrt{1+f^{1 / 2}+f}}{1-f^{1 / 2}}\right)+\sqrt{3} \arctan \left(\frac{\sqrt{3} f^{1 / 2}}{2+f^{1 / 2}}\right)\right] .
$$

\subsection{The explicit solutions for $f(\eta)$ and $\theta(\eta)$}

Based on the above mentioned asymptotic analysis for $f(\eta)$ and $\theta(\eta)$ and the homotopy analysis method (HAM), it is assumed that they can be expressed by a set of real functions in the following forms:

$$
\begin{aligned}
& f(\eta)=\sum_{k=0}^{\infty} f_{k}(\eta), \\
& \theta(\eta)=\sum_{k=0}^{\infty} \theta_{k}(\eta),
\end{aligned}
$$

where $f_{k}(\eta)$ and $\theta_{k}(\eta)$ are the high order deformation derivatives and are given by

$$
\begin{aligned}
& f_{k}(\eta)=\bar{A}_{k}^{0}+\sum_{j=1}^{4 k+4} \sigma_{k}^{j} \bar{A}_{k}^{j} \exp (-\lambda j \eta), \\
& \theta_{k}(\eta)=\sum_{j=1}^{4 k+4} \omega_{k}^{j} \bar{B}_{k}^{j} \exp (-\lambda j \eta),
\end{aligned}
$$

where $\lambda$ is a given positive constant, and $\sigma_{k}^{j}$ and $\omega_{k}^{j}$ are the coefficients defined as

$$
\sigma_{k}^{j}=\left\{\begin{array}{ll}
1, & 1 \leq j \leq 4 k+2, \\
0, & \text { other cases, }
\end{array} \quad \omega_{k}^{j}= \begin{cases}1, & 1 \leq j \leq 4 k-3, \\
0, & \text { other cases. }\end{cases}\right.
$$

The recursive coefficients $\bar{A}_{k}^{j}$ and $\bar{B}_{k}^{j}$ for $k \geq 1$ are determined by

$$
\begin{aligned}
& \bar{A}_{k}^{0}=0, \\
& \bar{A}_{k}^{1}=\chi_{k} \sigma_{k-1}^{1} \bar{A}_{k-1}^{1}+C_{1}^{k},
\end{aligned}
$$




$$
\begin{aligned}
& \bar{A}_{k}^{2}=\chi_{k} \sigma_{k-1}^{2} \bar{A}_{k-1}^{2}+C_{2}^{k}, \\
& \bar{A}_{k}^{3}=-\frac{\hbar_{f}}{6 \lambda^{3}}\left(\epsilon_{1} \bar{G}_{k}^{1}+\tilde{\mathcal{C}}_{k}^{1}\right), \\
& \bar{A}_{k}^{j}=\frac{\hbar_{f}}{\lambda^{3}(j-2)(j-1) j}\left(\epsilon_{1} \bar{G}_{k}^{j-2}+\tilde{\mathcal{C}}_{k}^{j-2}+\tilde{\mathcal{B}}_{k}^{j-2}+2 \tilde{\mathcal{A}}_{k}^{j-2}\right), \\
& \bar{B}_{k}^{1}=\chi_{k} \omega_{k-1}^{1} \bar{B}_{k-1}^{1}+C_{3}^{k}, \\
& \bar{B}_{k}^{2}=\chi_{k} \omega_{k-1}^{2} \bar{B}_{k-1}^{2}+\frac{1}{2 \lambda^{2}}\left(\frac{\epsilon_{2}}{\operatorname{Pr}} \bar{F}_{k}^{1}+\tilde{\mathcal{E}}_{k}^{1}\right), \\
& \bar{B}_{k}^{j}=\chi_{k} \omega_{k-1}^{j} \bar{B}_{k-1}^{j}+\frac{1}{\lambda^{2} j(j+1)}\left(\frac{\epsilon_{2}}{P r} \bar{F}_{k}^{j-1}+\tilde{\mathcal{E}}_{k}^{j-1}+\tilde{\mathcal{D}}_{k}^{j-1}\right),
\end{aligned}
$$

where $\hbar_{f}$ and $\hbar_{\theta}$ are the HAM auxiliary parameters; $C_{1}^{k}, C_{2}^{k}$, and $C_{3}^{k}$ are integral constants given by

$$
\begin{aligned}
C_{1}^{k}= & -\frac{\hbar_{f}}{6 \lambda^{3}}\left(\epsilon_{1} \bar{G}_{k}^{1}+\tilde{\mathcal{C}}_{k}^{1}\right)-\sum_{j=2}^{4 k+4} \frac{\hbar_{f}}{\lambda^{3}(j+1)(j+2)}\left(\epsilon_{1} \bar{G}_{k}^{j}+\tilde{\mathcal{C}}_{k}^{j}+\tilde{\mathcal{B}}_{k}^{j}+2 \tilde{\mathcal{A}}_{k}^{j}\right), \\
C_{2}^{k}= & \frac{\hbar_{f}}{3 \lambda^{3}}\left(\epsilon_{1} \bar{G}_{k}^{1}+\tilde{\mathcal{C}}_{k}^{1}\right)+\sum_{j=2}^{4 k+4} \frac{\hbar_{f}}{\lambda^{3} j(j+2)}\left(\epsilon_{1} \bar{G}_{k}^{j}+\tilde{\mathcal{C}}_{k}^{j}+\tilde{\mathcal{B}}_{k}^{j}+2 \tilde{\mathcal{A}}_{k}^{j}\right), \\
C_{3}^{k}= & -\frac{1}{\gamma+1} \sum_{j=2}^{4 k+4}\left[\frac{1}{\lambda j}+\frac{\gamma}{\lambda^{2} j(j+1)}\right]\left(\frac{\epsilon_{2}}{P r} \bar{F}_{k}^{j}+\tilde{\mathcal{E}}_{k}^{j}+\tilde{\mathcal{D}}_{k}^{j}\right) \\
& -\frac{1}{\gamma+1}\left(\frac{1}{\lambda}+\frac{\gamma}{2 \lambda^{2}}\right)\left(\frac{\epsilon_{2}}{P r} \bar{F}_{k}^{1}+\tilde{\mathcal{E}}_{k}^{1}\right),
\end{aligned}
$$

and

$$
\chi_{k}= \begin{cases}0, & k=1 \\ 1, & k>1\end{cases}
$$

Other coefficients appearing in the above recursive formulas are defined as

$$
\begin{array}{ll}
\bar{C}_{k}^{j}=(-\lambda j) \sigma_{k}^{j} \bar{A}_{k}^{j}, & \bar{E}_{k}^{j}=(\lambda j)^{2} \sigma_{k}^{j} \bar{A}_{k}^{j}, \quad \bar{G}_{k}^{j}=(-\lambda j)^{3} \sigma_{k}^{j} \bar{A}_{k}^{j}, \\
\bar{D}_{k}^{j}=(-\lambda j) \omega_{k}^{j} \bar{B}_{k}^{j}, & \bar{F}_{k}^{j}=(\lambda j)^{2} \omega_{k}^{j} \bar{B}_{k}^{j},
\end{array}
$$

and

$$
\begin{aligned}
& \tilde{\mathcal{A}}_{k}^{j}=\sum_{n=0}^{k-1} \sum_{s=\max \{1, j-4 n-4\}}^{\max \{4 k-4 n, j-1\}} \bar{C}_{k-1-n}^{s} \bar{C}_{n}^{j-s}, \\
& \tilde{\mathcal{B}}_{k}^{j}=\sum_{n=0}^{k-1} \sum_{s=\max \{1, j-4 n-4\}}^{\max \{4 k-4 n, j-1\}} \bar{A}_{k-1-n}^{s} \bar{E}_{n}^{j-s}, \\
& \tilde{\mathcal{C}}_{k}^{j}=\sum_{n=\left[\frac{j-1}{4}\right]}^{k-1} \bar{A}_{k-1-n}^{0} \bar{E}_{n}^{j},
\end{aligned}
$$




$$
\begin{aligned}
& \tilde{\mathcal{D}}_{k}^{j}=\sum_{n=0}^{k-1} \sum_{s=\max \{1, j-4 n-4\}}^{\max \{4 k-4 n, j-1\}} \bar{A}_{k-1-n}^{s} \bar{D}_{n}^{j-s}, \\
& \tilde{\mathcal{E}}_{k}^{j}=\sum_{n=\left[\frac{j-1}{4}\right]}^{k-1} \bar{A}_{k-1-n}^{0} \bar{D}_{n}^{j},
\end{aligned}
$$

where $[x]$ gives the greatest integer less than or equal to $x$.

When we set $\bar{A}_{0}^{0}=1, \bar{A}_{0}^{1}=-2, \bar{A}_{0}^{2}=1, \bar{B}_{0}^{1}=1, \bar{B}_{0}^{2}=-\lambda /(\gamma+2 \lambda)$, the purely explicit solutions for $f_{k}(\eta)$ and $\theta_{k}(\eta)(f(\eta)$ and $\theta(\eta))$ can be determined successively for $k=1,2,3, \ldots$ Note that here the homotopy auxiliary linear operators are selected as

$$
\mathcal{L}_{f}=\frac{\partial^{3}}{\partial \eta^{3}}+3 \lambda \frac{\partial^{2}}{\partial \eta^{2}}+2 \lambda^{2} \frac{\partial}{\partial \eta}, \quad \mathcal{L}_{\theta}=\frac{\partial^{2}}{\partial \eta^{2}}+\lambda \frac{\partial}{\partial \eta}
$$

and the homotopy auxiliary functions are chosen by

$$
\Omega_{f}(\eta)=\exp (-2 \lambda \eta), \quad \Omega_{\theta}(\eta)=\exp (-\lambda \eta)
$$

\section{Results and discussion}

We first examine the reliability and consistency of the explicit solution $f(\eta)$ denoted in Eq. (26). As shown in Figure 2, for various values of $\phi$, our explicit solution agrees well with the implicit one given in (25) in the whole range $0 \leq \eta<\infty$. Further, to check the

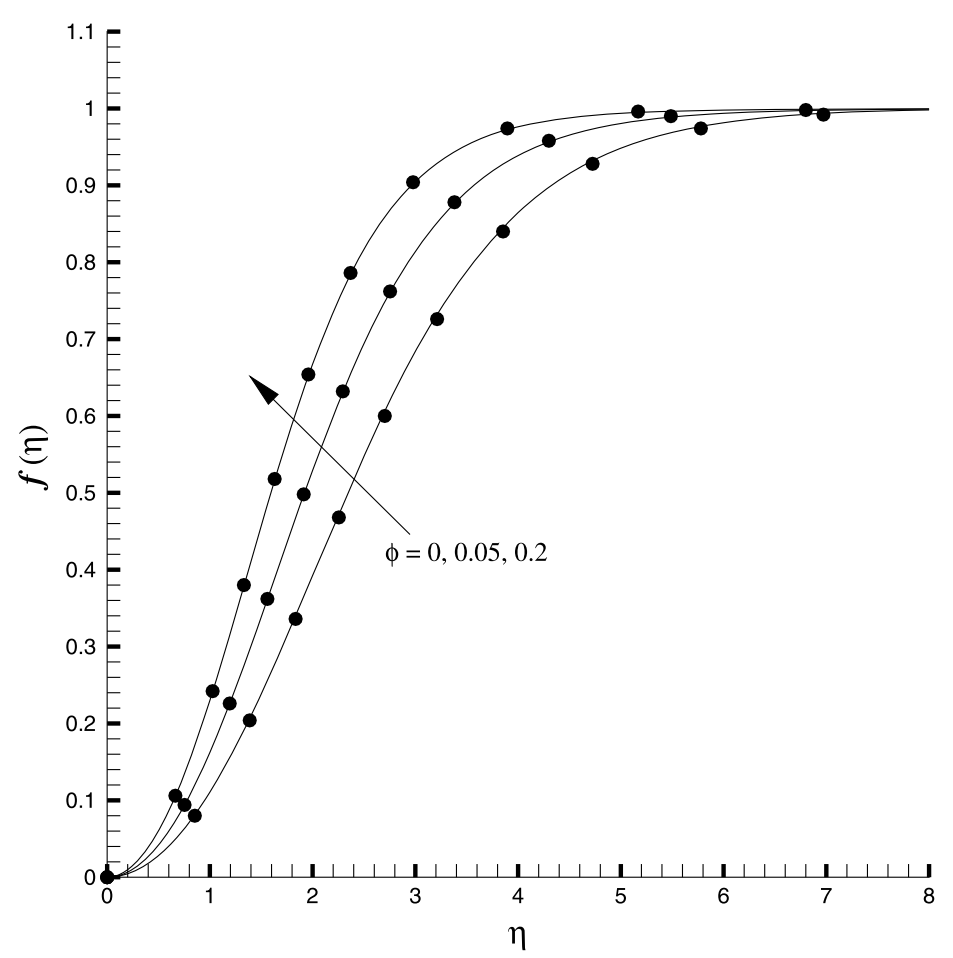

Figure 2 Comparison of the implicit solutions (25) with the explicit solutions at 100th order truncations for various values of $\phi$ in the case of $\hbar_{f}=-3 / 2$. Circle: the implicit solutions; line: the explicit solutions. 
Table 1 Computational errors for $\operatorname{Err}_{\theta}$ with $\hbar_{f}=-3 / 2$ and $\hbar_{\theta}=-1$ in the case of $\operatorname{Pr}=1$

\begin{tabular}{cll}
\hline $\boldsymbol{k}$ th order & $\boldsymbol{\phi}=\mathbf{2 0} / \mathbf{1 0 0}, \boldsymbol{\gamma = \mathbf { 1 }}$ & $\boldsymbol{\phi}=\mathbf{8} / \mathbf{1 0 0}, \boldsymbol{\gamma}=\mathbf{1 / 2}$ \\
\hline 0 & $8.18646 \times 10^{-3}$ & $4.04103 \times 10^{-3}$ \\
30 & $1.48150 \times 10^{-4}$ & $1.44346 \times 10^{-5}$ \\
60 & $6.73996 \times 10^{-5}$ & $4.55130 \times 10^{-6}$ \\
90 & $3.01452 \times 10^{-5}$ & $2.26475 \times 10^{-6}$ \\
120 & $2.74295 \times 10^{-5}$ & $1.37636 \times 10^{-6}$ \\
150 & $2.31459 \times 10^{-5}$ & $9.35413 \times 10^{-7}$ \\
180 & & $6.82566 \times 10^{-7}$ \\
\hline
\end{tabular}

The nanofluid is $\mathrm{Cu}$-water.

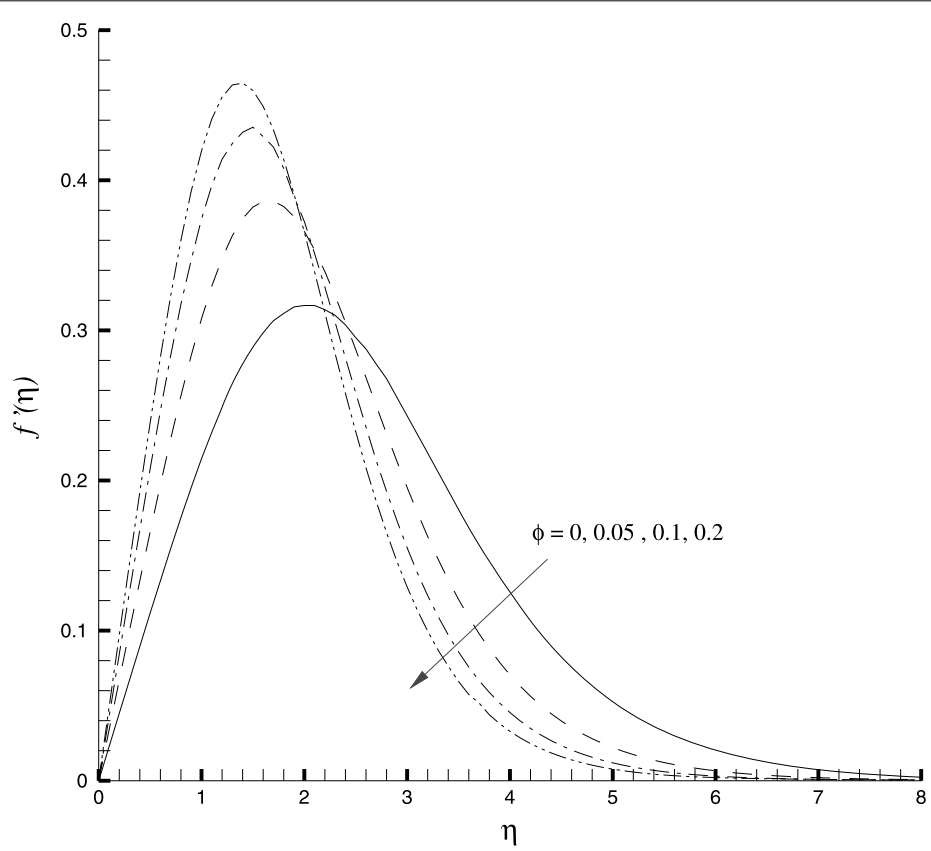

Figure 3 Velocity profile $f^{\prime}(\eta)$ for various values of $\phi$.

accuracy of the explicit solution $\theta(\eta)$, we define the following error estimation function:

$$
\operatorname{Err}_{\theta}=\lim _{\eta_{s} \rightarrow \infty} \int_{0}^{\eta_{s}}\left(\frac{\varepsilon_{2}}{\operatorname{Pr}} \theta^{\prime \prime}+f \theta^{\prime}\right)^{2} d \eta
$$

Substituting various orders of explicit solutions $f(\eta)$ and $\theta(\eta)$ into Eq. (44), the corresponding errors can be obtained, as listed in Table 1 . It is clearly seen from this table that the errors decrease monotonously with the increase of the computational order $k$ for both considered cases. The accuracies of those explicit solutions can be further improved when more and more orders of HAM truncations are involved.

The variation of the non-dimensional velocity profiles $f^{\prime}(\eta)$ with $\eta$ for various values of $\phi$ are plotted in Figure 3. It is found from this figure that for each selected value of $\phi$, there is a critical value of $\eta_{c}$ corresponding to the maximum velocity, when $\eta<\eta_{c}$, the velocity profile $f^{\prime}(\eta)$ increases dramatically as $\eta$ enlarges, while, when $\eta \geq \eta_{c}, f^{\prime}(\eta)$ decreases gradually as $\eta$ evolves. This is due to the fluid flow near the flat surface being restrained by the resting wall, while the flow at far field is retarded by the ambient quiescent fluid. On the 


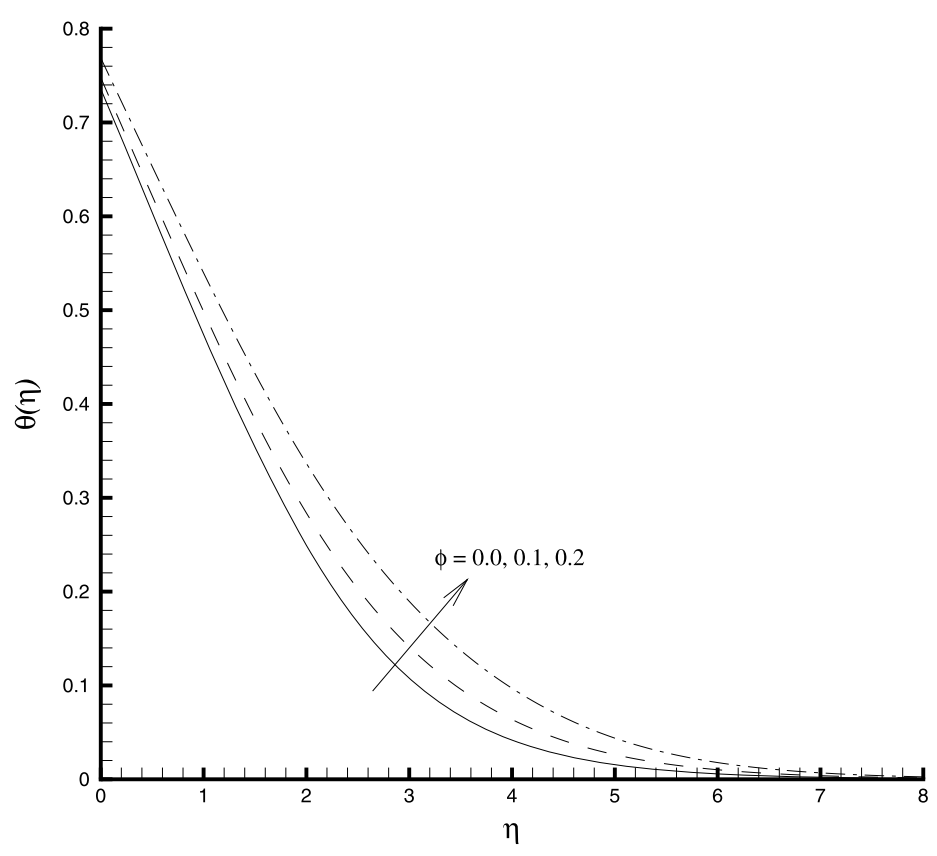

Figure 4 Temperature profiles $\theta(\eta)$ for various values of $\phi$ with $\operatorname{Pr}=1$ and $\gamma=1$.

other hand, we notice that the solid volumetric fraction parameter $\phi$ plays an important role in the variation of $f^{\prime}(\eta)$; the larger is the value of $\phi$, the greater is the maximum velocity $f^{\prime}(\eta)$. We further notice that the peak velocity of the flow increases with $\phi$ enlarging, but the critical value of $\eta_{c}$ varies contrarily, it decreases as $\phi$ increases. This can be explained by two reasons. One is that the higher concentration nanofluid flow possesses more kinetic energy than the lower concentration nanofluid or Newtonian fluid flows since they have the same incidence velocities. The other reason is that, unlike Newtonian fluid flows that are usually subject to a solid boundary, the nanofluid flows past a geometry are often restricted by a slip boundary, which is of help to reduce the flow drag near the resting wall.

We then consider the variation of the non-dimensional temperature profiles $\theta(\eta)$ with $\eta$ for various values of $\phi$ in the case of $\operatorname{Pr}=1$ and $\gamma=1$. As illustrated in Figure 4, $\theta(\eta)$ decreases continuously as $\eta$ evolves for any prescribed value of $\phi$. On the other hand, $\theta(\eta)$ enhances smoothly with $\phi$ increasing. This shows that the heat transfer characteristics of the considered fluid can be gradually improved as appropriate nanoparticles are continually added to it. We also notice that the reduced heat transfer parameter $\gamma$ has an obvious effect on $\theta(\eta)$. As shown in Figure 5, the temperature profile $\theta(\eta)$ increases consecutively as $\gamma$ enlarges for all $\eta$. This changing trend becomes more evident for small values of $\gamma$. We further notice that, as $\gamma$ tends to $0, \theta(\eta)$ approaches 0 as well. In this limiting case, there is no heat transfer by convection through the wall at all. As $\gamma$ tends to $\infty, \theta(\eta)$ approaches 1 . In this limiting case, the wall temperature is equal to the temperature of the fluid under the wall. Following that, we discuss the effect of the Prandtl number $P r$ on the variation of $\theta(\eta)$. As shown in Figure 6, $\theta(\eta)$ diminishes consecutively as $\operatorname{Pr}$ increases. This trend is very similar to the case of the Newtonian fluid flow, but the variation range for $\theta(\eta)$ is apparently smaller as compared with that of the Newtonian fluid flow. When $P r$ is sufficiently large, its effect on $\theta(\eta)$ becomes negligibly small. 


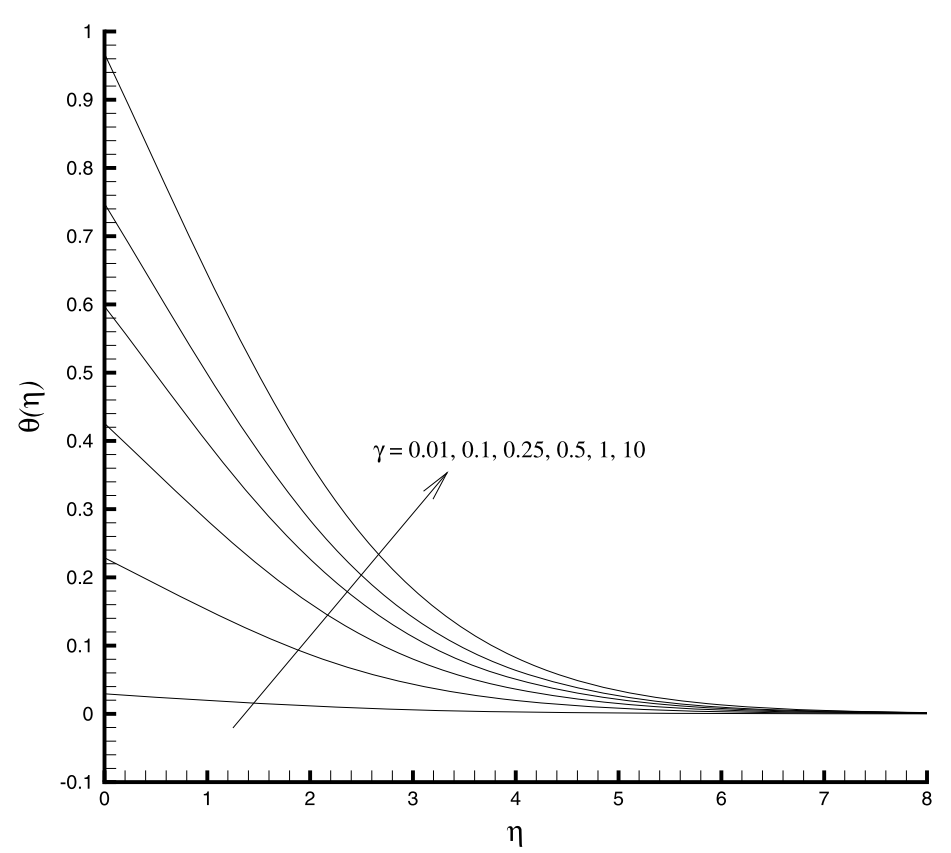

Figure 5 Temperature profiles $\theta(\eta)$ for different values of $\gamma$ with $\phi=0.1$ and $\operatorname{Pr}=1$.

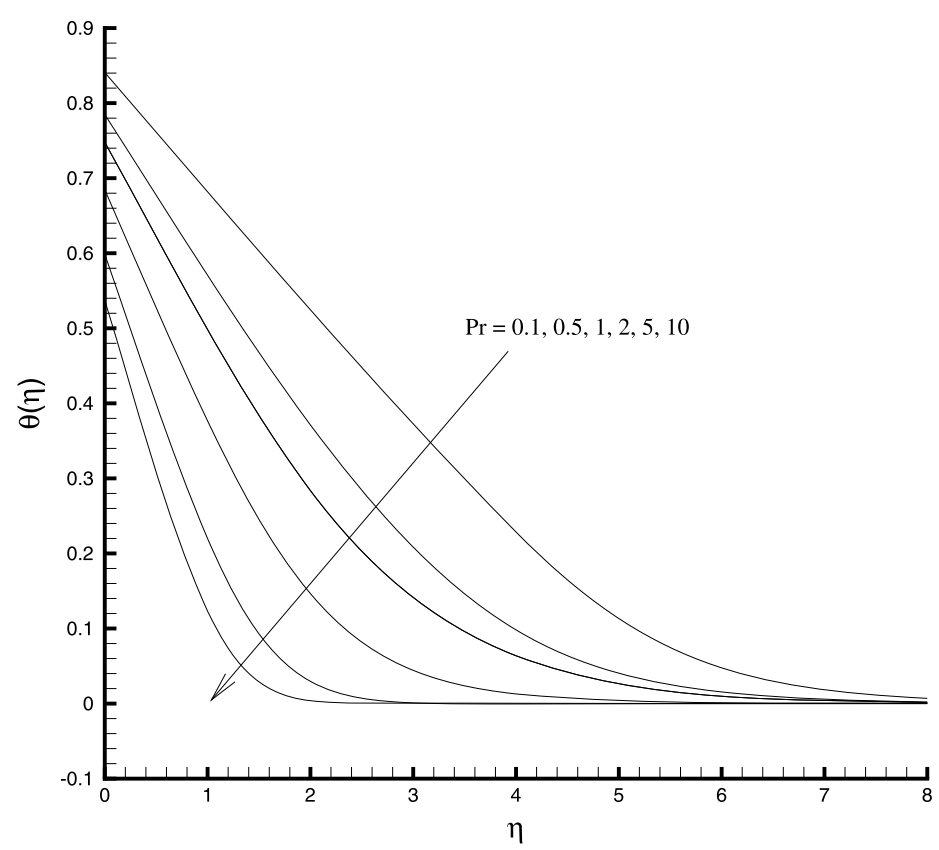

Figure 6 Temperature profiles $\theta(\eta)$ for different values of $\operatorname{Pr}$ with $\phi=0.1$ and $\gamma=1$. 


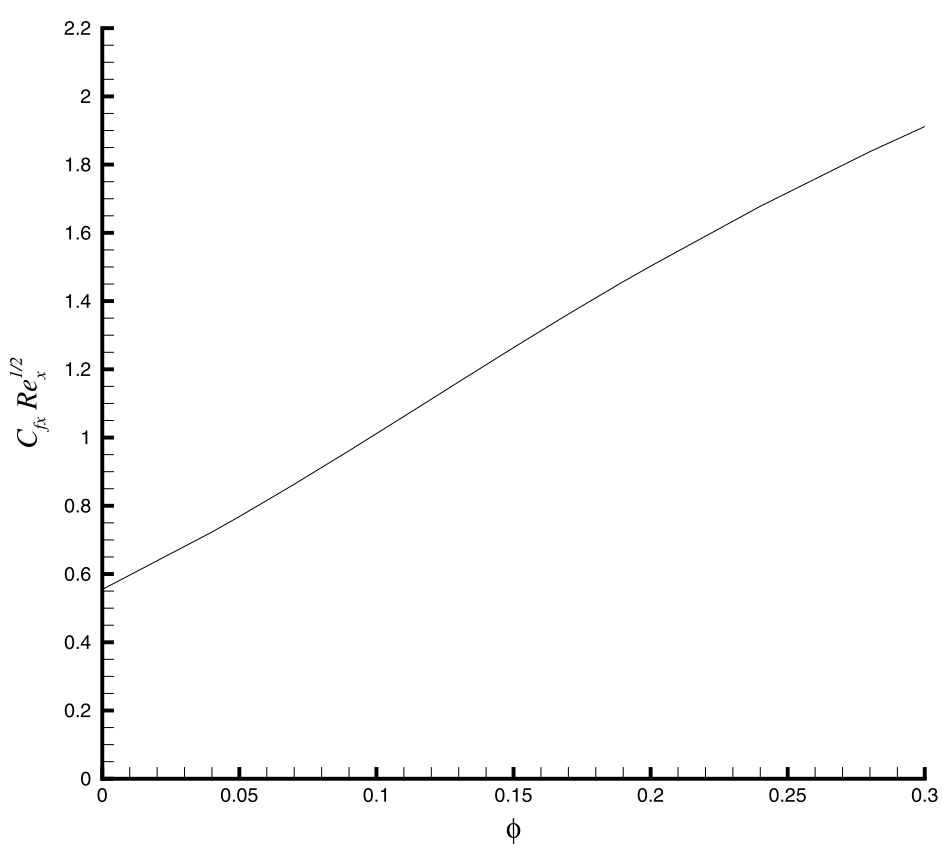

Figure 7 Variation of the reduced local skin friction coefficient $C_{f x}$ with $\phi$.

The local skin friction coefficient $C_{f x}$ and the local Nusselt number $N u_{x}$ are practically important in various applications regarding the flow and heat transfer in the boundary layer region. We therefore discuss them successively. As shown in Figure $7, C_{f x}$ enlarges almost linearly as $\phi$ evolves from 0 to 0.3 . Using the explicit solution for $f(\eta)$, one readily gives the explicit expression for $C_{f x} R e_{x}^{1 / 2}$, due to Eq. (12),

$$
C_{f x} R e_{x}^{1 / 2}=\frac{2}{(1-\phi)^{2.5}} \sum_{k=0}^{+\infty} \sum_{j=0}^{4 k+4}(\lambda j)^{2} \sigma_{k}^{j} \bar{A}_{k}^{j}
$$

Figure 8 gives the variation of the reduced Nusselt number $N u_{x} R e_{x}^{-1 / 2}$ with $\phi$ for $P r=1$ and $\gamma=1 / 10$. It is found that $N u_{x} R e_{x}^{-1 / 2}$ increases gradually with $\phi$ increasing, but its changing range is slower than that of $C_{f x} R e_{x}^{1 / 2}$. Though it is not shown in this paper, it is found that $\gamma$ has little effect on the variation of $N u_{x} R e_{x}^{-1 / 2}$, it is almost unchanged for all ranges $0<$ $\gamma<\infty$. Similarly, we are able to give the explicit expression for $N u_{x} R e_{x}^{-1 / 2}$ in the following form:

$$
N u_{x} R e_{x}^{-1 / 2}=-\frac{k_{n f}}{2 k_{f}}\left(\sum_{k=0}^{+\infty} \sum_{j=0}^{4 k+4}(-\lambda j) \omega_{k}^{j} \bar{B}_{k}^{j}\right) /\left(\sum_{k=0}^{+\infty} \sum_{j=0}^{4 k+4} \bar{B}_{k}^{j}\right) .
$$

The boundary layer thicknesses are of physical importance for this problem, defined by

$$
\delta_{f} / x=2 \eta_{f}^{\delta} / R e_{x}^{1 / 2}, \quad \delta_{\theta} / x=2 \eta_{\theta}^{\delta} / R e_{x}^{1 / 2},
$$

where $\delta_{f}$ is the thickness of the velocity boundary layer and $\delta_{\theta}$ is the thickness of the thermal boundary layer. Here it is assumed that the edges of the velocity and the thermal 


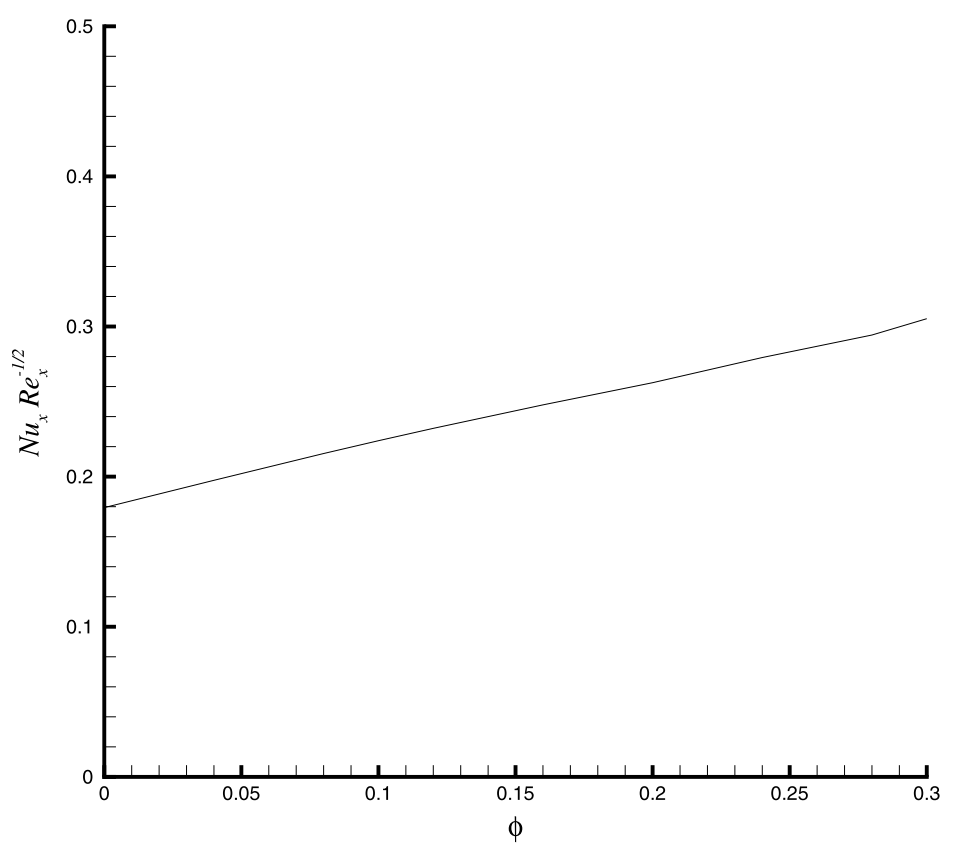

Figure 8 Variation of the reduced Nusselt number $N u_{x}$ with $\phi$ for $\gamma=1 / 10$ and $\operatorname{Pr}=1$.

boundary layers are located at the points $\eta_{f}^{\delta}$ and $\eta_{\theta}^{\delta}$ with $f^{\prime}\left(\eta_{f}^{\delta}\right)$ and $\theta\left(\eta_{\theta}^{\delta}\right)$ having the same value of 0.01 . The variations of the boundary layer thicknesses $\eta_{f}^{\delta}$ and $\eta_{\theta}^{\delta}$ with $\phi$ are plotted in Figure 9. It is seen from the figure that the $\eta_{f}^{\delta}$ increases continuously as $\phi$ enlarges from 0 to 0.24 ; beyond this value, its value decreases as $\phi$ evolves. $\eta_{\theta}^{\delta}$ exhibits a totally different trend, its values increase gradually as $\phi$ increases from 0 to 0.3 . But its increasing rate gradually descends as $\phi$ evolves, particularly, when $\phi \geq 0.24$, its effect on $\eta_{\theta}^{\delta}$ becomes dramatically small. It is worth mentioning that the homogeneous model is only valid for small values of $\phi$, i.e. $0 \leq \phi \leq 0.3$. When $\phi$ is considerably large, the nanofluid shows the characteristics of non-Newtonian fluids and this model cannot predict the behaviors of the nanofluid accurately.

\section{Conclusions}

In this paper, the laminar jet flow and heat transfer of a copper-water nanofluid over a resting wall has been examined in detail. By means of the homogeneous model, the original Navier-Stokes equations describing these jet flows have been reduced to a set of differential equations. An analytical solution for the velocity distribution has been obtained. The explicit solutions for both the velocity and the temperature distributions have been given by means of the HAM technique. The effects of the volumetric fraction parameter $\phi$ and the dimensionless heat transfer coefficient $\gamma$ on the velocity and temperature profiles, as well as on the reduced local skin friction coefficient and the reduced Nusselt number have been investigated. Some novel results and findings of this study have been presented:

1. An implicitly analytical solution for the velocity distribution is given, which has not been reported before.

2. A mathematical analysis for solution behavior at far field is presented. It is found that both the velocity and the temperature profiles decay exponentially at infinity. 


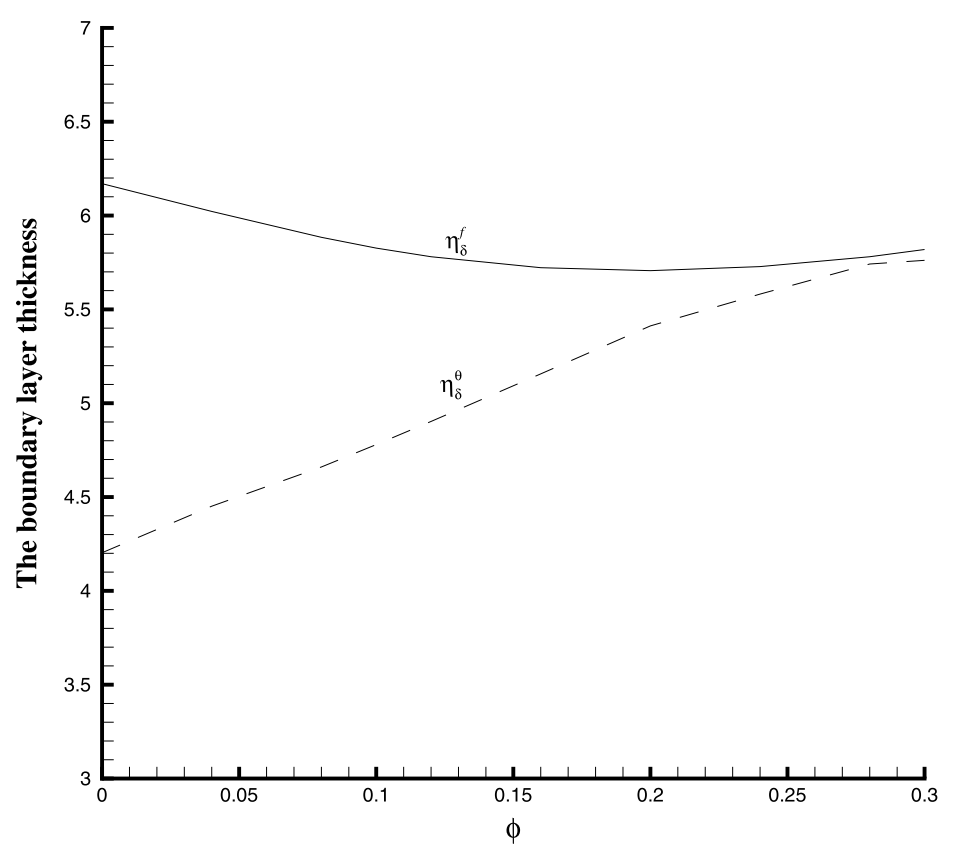

Figure 9 Variation of the boundary layer thicknesses of $\eta_{\delta}^{f}$ and $\eta_{\delta}^{\theta}$ with $\phi$ for $\gamma=1 / 10$ and $\operatorname{Pr}=1$.

3. Purely explicit solutions with high precision for both the velocity and the temperature distributions are obtained.

4. The volumetric fraction parameter $\phi$ has an important effect on the velocity and temperature distribution. The maximum velocity increases as $\phi$ enlarges. The temperature profiles increase as $\phi$ evolves, too. This means that the addition of nanoparticles into pure fluids is of help to reduce the flow drag near the wall and to improve the heat transfer capability of the base fluids.

5. The dimensionless heat transfer parameter $\gamma$ plays a key role on the variation of the temperature profiles. The temperature profiles enhances as $\gamma$ increases.

\section{Competing interests}

The authors declare that they have no competing interests.

Authors' contributions

All authors contributed equally to the writing of this paper. All authors read and approved the final manuscript.

\section{Author details}

'State Key Lab of Ocean Engineering, School of Naval Architecture, Ocean and Civil Engineering, Shanghai Jiao Tong University, Shanghai, 200240, China. ${ }^{2}$ Department of Computer Science and Engineering, Shanghai Jiao Tong University, Shanghai, 200240, China.

\section{Acknowledgements}

We extend our sincere appreciations to the Program for New Century Excellent Talents in University (Grant No. NCET-12-0347), and to the Program of Innovative Fundings for Youth of the State Key Laboratory of Ocean Engineering (Shanghai Jiao Tong University) (Grant No. GKZD010059-17) for their financial supports.

Received: 13 December 2013 Accepted: 15 April 2014 Published: 1 September 2014

\section{References}

1. Glauert, MB: The wall jet. J. Fluid Mech. 16, 625-643 (1956)

2. Merkin, JH, Needham, DJ: A note on the wall-jet problem. J. Eng. Math. 20, 21-26 (1986)

3. Merkin, JH, Needham, DJ: A note on the wall-jet problem II. J. Eng. Math. 21, 17-22 (1987)

4. Magyari, E, Keller, B: The algebraically decaying wall jet. Eur. J. Mech. B, Fluids 23, 601-605 (2004) 
5. Cohen, J, Amitay, M, Bayly, BJ: Laminar-turbulent transition of wall jet flows subjected to blowing and suction. Phys. Fluids A 4, 283-289 (1992)

6. Xu, H, Liao, SJ, Wu, GX: A family of new solutions on the wall jet. Eur. J. Mech. B, Fluids 27, 322-334 (2008)

7. Magyari, E, Keller, B, Pop, I: Heat transfer characteristics of a boundary-layer flow driven by a power-law shear over a semi-infinite flat plate. Int. J. Heat Mass Transf. 47, 31-34 (2004)

8. Cossali, GE: Similarity solutions of energy and momentum boundary layer equations for a power-law shear driven flow over a semi-infinite flat plate. Eur. J. Mech. B, Fluids 25, 18-32 (2006)

9. Fan, $\mathrm{T}, \mathrm{Xu}, \mathrm{H}$ : New branches with algebraical behaviour for thermal boundary-layer flow over a permeable sheet. Commun. Nonlinear Sci. Numer. Simul. 18, 1162-1174 (2013)

10. Choi, SUS: Enhancing thermal conductivity of fluids with nanoparticle. In: The Proceedings of the 1995 ASME International Mechanical Engineering Congress and Exposition, ASME, FED 231/ MD66, San Francisco, USA, November 12-17, pp. 99-105 (1995)

11. Maïga, SEB, Palm, SJ, Nguyen, CT, Roy, G, Galanis, N: Heat transfer enhancement by using nanofluids in forced convection flows. Int. J. Heat Fluid Flow 26(4), 530-546 (2005)

12. Xuan, YM, Roetzel, W: Conceptions for heat transfer correlation of nanofluids. Int. J. Heat Mass Transf. 43(19), 3701-3707 (2000)

13. Buongiorno, J: Convective transport in nanofluids. J. Heat Transf. 128(3), 240-250 (2006)

14. Buongiorno, J, Hu, W: Nanofluid coolants for advanced nuclear power plants. In: Proceedings of ICAPP '05, Paper no. 5705, Seoul, May 15-19 (2005)

15. Trisaksri, V, Wongwises, S: Critical review of heat transfer characteristics of nanofluids. Renew. Sustain. Energy Rev. 11, 512-523 (2007)

16. Wang, XQ, Mujumdar, AS: Heat transfer characteristics of nanofluids: a review. Int. J. Therm. Sci. 46, 1-19 (2007)

17. Eastman, JA, Phillpot, SR, Choi, SUS, Keblinski, P. Thermal transport in nanofluids. Annu. Rev. Mater. Res. 34, 219-246 (2004)

18. Kakac, S, Pramaumjaroenkij, A: Review of convective heat transfer enhancement with nanofluids. Int. J. Heat Mass Transf. 52, 3187-3196 (2009)

19. Bachok, N, Ishak, A, Pop, l: Flow and heat transfer over a rotating porous disk in a nanofluid. Physica B 406, 1767-1772 (2011)

20. Rohni, AM, Ahmad, S, Pop, I: Flow and heat transfer over an unsteady shrinking sheet with suction in nanofluids. Int. J. Heat Mass Transf. 55, 1888-1895 (2012)

21. Yacob, NA, Ishak, A, Pop, I: Falkner-Skan problem for a static or moving wedge in nanofluids. Int. J. Therm. Sci. 50, 133-139 (2011)

22. Vajravelu, K, Prasad, KV, Lee, J, Lee, C, Pop, I, Vab Gorder, RA: Convective heat transfer in the flow of viscous Ag-water and Cu-water nanofluids over a stretching surface. Int. J. Therm. Sci. 50, 843-851 (2011)

23. Aziz, A: A similarity solution for laminar thermal boundary layer over a flat plate with a convective surface boundary condition. Commun. Nonlinear Sci. Numer. Simul. 14, 1064-1068 (2009)

24. Ishak, A: Similarity solutions for flow and heat transfer over a permeable surface with convective boundary condition. Appl. Math. Comput. 217, 837-842 (2010)

25. Aziz, A, Khan, WA: Natural convective boundary layer flow of a nanofluid past a convectively heated vertical plate. Int J. Therm. Sci. 52, 83-90 (2012)

26. Makinde, OD, Aziz, A: MHD mixed convection from a vertical plate embedded in a porous medium with a convective boundary condition. Int. J. Therm. Sci. 49, 1813-1820 (2010)

27. Hayat, T, Iqbal, Z, Qasim, M, Obaidat, S: Steady flow of an Eyring Powell fluid over a moving surface with convective boundary conditions. Int. J. Heat Mass Transf. 55, 1817-1822 (2012)

28. Brinkman, HC: The viscosity of concentrated suspensions and solutions. J. Chem. Phys. 20, 571-581 (1952)

29. Xuan, Y, Li, Q: Investigation on convective heat transfer and flow features of nanofluids. J. Heat Transf. 125, 151-155 (2003)

doi:10.1186/1687-2770-2014-163

Cite this article as: Raees et al.: Explicit solutions of wall jet flow subject to a convective boundary condition.

Boundary Value Problems 2014 2014:163.

\section{Submit your manuscript to a SpringerOpen ${ }^{\circ}$ journal and benefit from:}

- Convenient online submission

Rigorous peer review

- Immediate publication on acceptance

- Open access: articles freely available online

- High visibility within the field

- Retaining the copyright to your article 